\title{
Anomalous Electron Heat Transport Driven by Low-Frequency Electromagnetic Turbulence
}

\author{
A. A. Thoul, P. L. Similon, and R. N. Sudan \\ Laboratory of Plasma Studies, Cornell University, Ithaca, New York 14853
}

(Received 5 August 1987)

\begin{abstract}
We consider the anomalous electron and heat transport in a tokamak plasma. The electrons are described by the nonlinear drift-kinetic equation. We analyze transport through the averaged response function in the presence of drift-Alfvén wave turbulence. In contrast to recent findings by Terry, Diamond, and Hahm, we conclude that magnetic fluctuations lead to a substantial transport of both parallel and perpendicular energies. The latter, previously neglected, is found to be significant and of the order of the test-particle diffusion.
\end{abstract}

PACS numbers: 52.35.Ra, 47.25.Jn, 52.35.Kt, 52.35.Qz

Observations in large tokamak machines ${ }^{1}$ have shown that electron heat transport is a major energy-loss mechanism, and is 2 orders of magnitude larger than what the classical diffusion theory predicts. It is believed that magnetic turbulence is an important candidate to explain anomalous transport in a tokamak plasma. ${ }^{2}$

Terry, Diamond, and $\mathrm{Hahm}^{3}$ have recently attempted to improve the quasilinear models ${ }^{4}$ and have included in their study the self-consistency of the particle evolution and the fields. Constraints appear through quasineutrality and Ampère's law. They reach the conclusion that the magnetic fluctuations do not contribute to the relaxation of the average electron distribution function, and therefore do not contribute to the radial transport of any velocity moment.

In this Letter we show that while electron transport is indeed determined by ion transport because of quasineutrality, there can nevertheless be a significant electron parallel and perpendicular heat transport, in contrast to the conclusion of Ref. 3. Electrons diffuse because of resonant processes such as Compton scattering. Because of the resonance condition $v^{2} \|_{\text {res }}^{2}=\left(\omega / k_{\|}\right)^{2} \ll v_{e}^{2}$, where $v_{e}^{2}=T / m_{e}$, these particles have small parallel energy. They create self-consistent electromagnetic fields in response to which other electrons are exchanged to main- tain quasineutrality. However, these other electrons may have $v^{2}{ }^{2}$ of order $v_{e}^{2}$, because they are not constrained to be resonant. This explains how, in spite of ambipolarity, there can be transport of electron parallel energy. The reasoning above applies equally for the transport of perpendicular energy. It will be shown that the particle dynamics is independent of $v_{\perp}$; therefore the transport of perpendicular energy is of the order of the test-particle diffusion.

We consider a slab geometry with density and temperature gradients, and without magnetic shear. The magnetic field is along the $z$ direction, the gradients are along the (radial) $x$ direction, and $y$ is the (poloidal) ignorable coordinate. The electrons are governed by the drift-kinetic equation in which we keep the nonlinear $\mathbf{E} \times \mathbf{B}$ drift, nonlinear magnetic flutter, and nonlinear parallel acceleration, but we neglect the diffusion in velocity space. The ion dynamics is linearized and depends on the electrostatic potential $\tilde{\phi}$ only, with a constant of proportionality $v$. The ion current is neglected, and nonlinear ion response ${ }^{5}$ is not considered here. The plasma is thus described by the drift-kinetic equation, the quasineutrality condition, Ampère's law, and the relation between the perturbed ion density $\tilde{n}_{i}$ and the electrostatic potential $\tilde{\phi}$, in Fourier representation $k \equiv(\mathbf{k}, \omega)$ :

$$
\begin{aligned}
& -i\left(\omega-k_{\left\|c^{\prime}\right\|}\right) \tilde{h}_{k}-i\left(\omega-\omega_{*}\right) f_{0}\left(v^{\prime}\right) F_{0}\left(v_{\perp}^{2}\right) \frac{e}{T}\left(\tilde{\phi}-\frac{v_{\|}}{c} \tilde{A}_{\|}\right)_{k}=\sum_{k^{\prime}+k^{\prime \prime}=k} w_{k^{\prime} k^{\prime \prime}}\left(\tilde{\phi}-\frac{v_{\|}}{c} \tilde{A}_{\|}\right)_{k^{\prime}} \tilde{h}_{k^{\prime \prime}}, \\
& \int d^{3} v^{\prime} \tilde{h}_{k}+\left(1-v_{k}\right) \frac{e}{T} \tilde{\phi}_{k}=0 \\
& \int d^{3} v^{\cdot} \frac{v^{\prime} \|}{c} \tilde{h}_{k}+\frac{k^{2}}{k_{\mathrm{D} e}^{2}} \frac{e}{T} \tilde{A}_{\| k}=0 \\
& \tilde{n}_{i}=n_{0} v_{k} \frac{e}{T} \tilde{\phi}_{k}
\end{aligned}
$$

where $\tilde{h}\left(v_{\|}\right)$is the nonadiabatic part of the perturbed electron distribution function, $\|$and $\perp$ refer to components parallel and perpendicular to the equilibrium magnetic field $\mathbf{B}_{0}=B \hat{\mathbf{b}}_{0}$, and $\tilde{\phi}$ and $\tilde{A}_{\|}$are the electrostatic and vector potentials. The nonlinear coupling coefficient is $w_{k^{\prime} k^{\prime \prime}}=(c / B) \hat{\mathbf{b}}_{0} \cdot\left(\mathbf{k}^{\prime} \times \mathbf{k}^{\prime \prime}\right)$; the diamagnetic frequency is given by $\omega_{*}\left(v_{\|}, v_{\perp}^{2}\right)=\omega_{*}^{n}+\omega_{*}^{T}\left(v^{\prime}{ }^{2}+v_{\perp}^{2}-3 v_{e}^{2}\right) / 2 v_{e}^{2}$, where $\omega_{*}^{n}=-(c T / e B) \mathbf{k} \cdot \hat{\mathbf{b}}_{0} \times\left(\nabla n_{0} / n_{0}\right)$ and $\omega_{*}^{T}=-(c T / e B) \mathbf{k} \cdot \hat{\mathbf{b}}_{0} \times(\nabla T / T) ;$ the local Maxwellian distribution function is given by $f_{0}\left(v_{\|}\right)=\left(2 \pi v_{e}^{2}\right)^{-1 / 2} \exp \left(-v_{\|}^{2} / 2 v_{e}^{2}\right)$ and $F_{0}\left(v_{\perp}^{2}\right)=\left(2 \pi v_{e}^{2}\right)^{-1}$ 
$\times \exp \left(-v_{\perp}^{2} / 2 v_{e}^{2}\right)$; and $k_{\mathrm{De}}$ is the Debye wave number.

We wish to study the transport of both parallel and perpendicular energy, because whereas the resonance condition $v_{\| \text {res }}^{2}=\left(\omega / k_{\|}\right)^{2} \ll v_{e}^{2}$ may limit $v_{\|}^{2}$, no such limitation exists on $v_{\perp}^{2}$. Considerable simplification occurs because the $v_{\|}$ and $v_{\perp}$ dependence in Eqs. (1)-(3) can easily be separated, if the nonadiabatic perturbation of the distribution function is written as

$$
\tilde{h}\left(v_{\|}, v_{\perp}^{2}\right)=\tilde{p}\left(v_{\|}\right) F_{0}\left(v_{\perp}^{2}\right)+\tilde{q}\left(v_{\|}\right) F_{0}\left(v_{\perp}^{2}\right)\left(v_{\perp}^{2}-2 v_{e}^{2}\right) / 2 v_{e}^{2} .
$$

Equation (1) then splits naturally into two equations, one each for $\tilde{p}_{k}$ and $\tilde{q}_{k}$, both independent of $v_{\perp}^{2}$ :

$$
\begin{aligned}
& -i\left(\omega-k_{\| v_{\|}}\right) \tilde{p}_{k}+b_{k}\left(v_{\|}\right) \frac{e}{T}\left(\tilde{\phi}-\frac{v^{\prime} \|}{c} \tilde{A}_{\|}\right)_{k}=\sum_{k^{\prime}+k^{\prime \prime}=k} w_{k^{\prime} k^{\prime \prime}}\left(\tilde{\phi}-\frac{v_{\|}}{c} \tilde{A}_{\|}\right)_{k^{\prime}} \tilde{p}_{k^{\prime \prime}}+\xi_{k}\left(v_{\|}\right), \\
& -i\left(\omega-k_{\| v_{\|}}\right) \tilde{q}_{k}+\beta_{k}\left(v_{\|}\right) \frac{e}{T}\left(\tilde{\phi}-\frac{v^{\prime} \|}{c} \tilde{A}_{\|}\right)_{k}=\sum_{k^{\prime}+k^{\prime \prime}=k} w_{k^{\prime} k^{\prime \prime}}\left(\tilde{\phi}-\frac{v_{\|}}{c} \tilde{A}_{\|}\right)_{k^{\prime}} \tilde{q}_{k^{\prime \prime}}+\lambda_{k}\left(v_{\|}\right),
\end{aligned}
$$

where $b_{k}=-i\left[\omega-\omega_{*}^{n}-\omega_{*}^{T}\left(v_{\|}^{2}-v_{e}^{2}\right) / 2 v_{e}^{2}\right] f_{0}\left(v_{\|}\right)$and $\beta_{k}=i \omega_{*}^{T} f_{0}\left(v_{\|}\right)$. Arbitrary external source terms $\xi_{k}$ and $\lambda_{k}$ have been introduced here in order to study how the plasma responds to external perturbations (i.e., its relaxation); from this, the transport properties of the configuration can be deduced.

Notice that $\tilde{q}_{k}$ does not appear in Eqs. (2) and (3), so that $\tilde{p}_{k}, \tilde{\phi}_{k}$, and $\tilde{A}_{\| k}$ form a closed system. The potentials do not depend on $\tilde{q}_{k}$, which is only passively convected according to (6).

We study the transport through the mean evolution of a given perturbation in the turbulent field, via the average response function computed at long wavelengths, i.e., at small $k_{0}$ (somewhat related discussion has been made by Krommes, Oberman, and Kleva ${ }^{6}$ ). The objective of the calculation is to determine the nonlinear fluxes in response to arbitrary sources $\xi_{k_{0}}$ and $\lambda_{k_{0}}$ in a background of turbulent drift-Alfvén fluctuations. To obtain the total fluxes across the magnetic surfaces, we calculate them at $k_{0 \|}=k_{0 y}=0$, which corresponds to integrating them over the magnetic surface. The average of the nonlinear terms in the right-hand sides of Eqs. (5) and (6) is denoted by $\Xi$ and $\Lambda$, respectively, and is the divergence of the nonlinear fluxes. The radial fluxes of particle, parallel energy, and perpendicular energy are then given by

$$
\begin{aligned}
& \Gamma_{n}=-\left(i k_{0 x}\right)^{-1} n_{0} \int d v_{\|} \Xi^{k_{0}}, \\
& \Gamma_{H_{\|}}=-\left(i k_{0 x}\right)^{-1} n_{0} \int d v_{\|} v_{\|}^{2} \Xi_{k_{0}}, \\
& \Gamma_{H_{\perp}}=-\left(i k_{0 x}\right)^{-1} n_{0} 2 v_{e}^{2}\left[\int d v_{\|} \Xi_{k_{0}}+\int d v_{\|} \Lambda_{k_{0}}\right] .
\end{aligned}
$$

The first term in Eq. (9) represents the heat transport as if it were simply given by the particle flux times the thermal energy, and the second term represents the correction due to the non-Maxwellian nature of the perturbed distribution function. The fluxes $\Gamma$ calculated here are the flux increments coming from an increase in temperature and density gradients. The equilibrium fluxes are obtained by integration of $\Gamma$ as the gradients are raised from zero to their equilibrium values. To obtain the response to a perturbation in density $\delta n_{k_{0}}$ (for both the ions and the electrons) or temperature $\delta T_{\| k_{0}}$ or $\delta T_{\perp k_{0}}$, we choose as our source term a low-frequency $\left(\omega_{0}\right)$ perturbation of a Maxwellian distribution:

$$
\xi_{k_{0}}\left(v_{\|}\right)=-i \omega_{0}\left(\delta n_{k_{0}} / n_{0}\right) f_{0}\left(v_{\|}\right)-i \omega_{0}\left(\delta T_{\| k_{0}} / T\right) f_{0}\left(v_{\|}\right)\left(v_{\|}^{2}-v_{e}^{2}\right) / 2 v_{e}^{2},
$$

and

$$
\lambda_{k_{0}}\left(v_{\|}\right)=-i \omega_{0}\left(\delta T_{\perp k_{0}} / T\right) f_{0}\left(v_{\|}\right) .
$$

The average response functions $\langle\delta \hat{p} / \delta \xi\rangle$ and $\langle\delta \hat{q} / \delta \lambda\rangle$ (where $\hat{p}$ and $\hat{q}$ are the responses to the perturbations $\xi$ and $\lambda$, distinct from the fluctuating quantities $\tilde{p}$ and $\tilde{q}$ ) are then given to the linear approximation by $\hat{p}_{k_{0}} \approx \xi_{k_{0}}$ l $\left(-i \omega_{0}\right)-(e / T) \hat{\phi}_{k_{0}} f_{0}$ and $\hat{q}_{k_{0}} \approx \lambda_{k_{0}} /\left(-i \omega_{0}\right)$. The corrections due to the nonlinear relaxation are evaluated here with the direct-interaction approximation (DIA) in the renormalized weak-turbulence limit. As a result, we find that $\Xi$ can be written as a linear function of $\hat{p}_{k}, \tilde{\phi}_{k}$, and $\hat{A}_{\| k}$, and $\Lambda$ as a linear function of $\hat{q}_{k}, \hat{p}_{k}, \hat{\phi}_{k}$, and $\hat{A}_{\| k \cdot}{ }^{7,8}$ Note that the following conservation laws are verified by the exact equation: $\sum_{k}\left[\tilde{\phi}-\left(v_{\|} / c\right) \tilde{A}_{\|}\right]_{k}^{*} \Xi_{k}=0$ and $\sum_{k}\left[\tilde{\phi}-\left(v_{\|} / c\right) \tilde{A}_{\|}\right]_{k}^{*} \Lambda_{k}=0, \forall v_{\|}$, which means that in a reference frame moving with the parallel velocity of the particle the drift is perpendicular to both the local electric field and the magnetic field. This property is preserved by the DIA statistical closure.

When $\tilde{n}_{i}$ is not in phase with $\tilde{\phi}$, e.g., when the ions are dissipative, the particle flux is ambipolar and regulated by the ion response. What we wish to emphasize here is more apparent if we take $\tilde{n}_{i}$ in phase with $\tilde{\phi}$, i.e., if $\operatorname{Im} v=0$. In this case the ions cannot diffuse, and because of ambipolarity the electrons do not diffuse either. We find that although $\Gamma_{n}$ vanishes for $k_{0}$ small, generally $\Gamma_{H_{\|}}$and $\Gamma_{H_{\perp}}$ do not vanish. We can illustrate the cancellations occurring in the calculation of the particle flux by 
choosing a few terms in $\Xi$. Define $a_{k}=1-v_{k}, d_{k}=k^{2} / k_{\mathrm{D} e}^{2}$, the propagator $g_{k}=\left(-i \omega+i k_{\| v_{\|}}\right)^{-1}$, and the dispersion function

$$
G_{k}^{-1}=\left[\left(a_{k}-\int d v_{\|} g_{k} b_{k}\right)\left(d_{k}+\int d v_{\|} g_{k} b_{k} v^{2}{ }^{2} / c^{2}\right)+\left(\int d v^{\prime} g_{k} b_{k} v_{\|} / c\right)^{2}\right] .
$$

Then the approximate expression of $\Xi$ provided by the DIA closure is

$$
\begin{aligned}
& \Xi_{k}=-\sum_{k^{\prime}+k^{\prime \prime}=k}\left|w_{k^{\prime} k^{\prime \prime}}\right|^{2}\left\langle\left|\tilde{A}_{\|}\right|^{2}\right\rangle_{k^{\prime \prime}} \\
& \times\left\{\hat{p}_{k} g_{k^{\prime}} \cdot \frac{v^{\prime} \|^{2}}{c^{2}}+G_{k^{\prime}} g_{k}, b_{k}, \frac{v^{\prime} \|}{c}\left[\left(d_{k^{\prime}}+\int d v^{\prime} \| g_{k^{\prime}}, b_{k^{\prime}} \frac{v^{2} \|}{c^{2}}\right)\left(\int d v^{\prime} g_{k^{\prime}}, \hat{p}_{k} \frac{v^{\prime} \|}{c}\right)\right.\right. \\
& \left.-\left(\int d v^{\prime} g_{k^{\prime}}, b_{k^{\prime}}, \frac{v^{\prime} \|}{c}\right)\left(\int d v^{\prime} g_{k^{\prime}}, \hat{p}_{k} \frac{v^{2} \|^{2}}{c^{2}}\right)\right]-G_{k^{\prime}} g_{k^{\prime}}, b_{k^{\prime}}, \frac{v^{2}}{c^{2}}\left[\left(a_{k^{\prime}}-\int d v_{\|} g_{k^{\prime}} b_{k^{\prime}}\right)\left(\int d v_{\|} g_{k^{\prime}} \hat{p}_{k} \frac{v_{\|}^{2}}{c^{2}}\right)\right. \\
& \left.\left.+\left(\int d v_{\|} g_{k}, b_{k}, \frac{v_{\|}}{c}\right)\left(\int d v_{\|} g_{k}, \hat{p}_{k} \frac{v_{\|}}{c}\right)\right]\right\} \\
& -\sum_{k^{\prime}+k^{\prime \prime}=k} \frac{T}{e}\left|w_{k^{\prime} k^{\prime \prime}}\right|^{2}\left\langle\tilde{A}_{\|}^{*} \tilde{p}\right\rangle_{k^{\prime \prime}} \\
& \times\left\{G_{k^{\prime}} \frac{v^{\prime} \|}{c}\left[\left(a_{k^{\prime}}-\int d v_{\|} g_{k^{\prime}}, b_{k^{\prime}}\right)\left(\int d v_{\|} g_{k^{\prime}} \hat{p}_{k} \frac{v^{\prime} \|^{2}}{c^{2}}\right)+\left(\int d v_{\|} g_{k^{\prime}}, b_{k^{\prime}} \frac{v_{\|}}{c}\right)\left(\int d v_{\|} g_{k^{\prime}} \hat{p}_{k} \frac{v_{\|}}{c}\right)\right]\right\}+\ldots .
\end{aligned}
$$

If we integrate this expression over $v^{\prime} \|$ at $k=k_{0}$ to get the particle flux, it is easy to see how the cancellations occur by multiplying the first term in the curly brace by $G_{k^{\prime}} G_{k^{\prime}}^{-1}$. With use of Ampère's law, all these terms cancel out, in the limit of small $k_{0 x}$. More explicitly, the range of $k_{0 x}$ is determined by the inequalities $\left|\nabla n_{0} / n_{0}\right|,|\nabla T / T| \ll k_{0 x} \ll k_{x}^{\prime \prime}$, where $k_{x}^{\prime \prime}$ represents a typical wave number of the turbulence. In other words, ambipolarity is achieved when the flux is averaged over the spatial extent of the modes. ${ }^{9}$ The dots in Eq. (12) represent other similar terms which cancel out in the same manner.

When Eq. (12) is multiplied by $v_{\|}^{2}$ and then integrated over $v_{\|}$to get the parallel energy flux, these cancellations do not occur, showing that the transport of parallel energy does not vanish in general. The result for $\Lambda$ is similar to the result for $\Xi$, and the corresponding terms are

$$
\begin{aligned}
& \Lambda_{k}=-\sum_{k^{\prime}+k^{\prime \prime}=k}\left|w_{k^{\prime} k^{\prime \prime}}\right|^{2}\left\langle\left|\tilde{A}_{\|}\right|^{2}\right\rangle_{k^{\prime \prime}} \\
& \times\left\{\hat{q}_{k} g_{k}, \frac{v_{\|}^{2}}{c^{2}}+G_{k}, g_{k}, \beta_{k^{\prime}} \cdot \frac{v^{\prime} \|}{c}\left[\left(d_{k^{\prime}}+\int d v_{\|} g_{k}, b_{k}, \frac{v_{\|}^{2}}{c^{2}}\right)\left(\int d v_{\|} g_{k}, \hat{p}_{k} \frac{v_{\|}}{c}\right)\right.\right. \\
& \left.-\left(\int d v^{\prime} g_{k^{\prime}}, b_{k^{\prime}}, \frac{v_{\|}}{c}\right)\left(\int d v{ }_{\|} g_{k}, \hat{p}_{k} \frac{v_{\|}^{2}}{c^{2}}\right)\right]-G_{k^{\prime}} g_{k}, \beta_{k^{\prime}}, \frac{v_{\|}^{2}}{c^{2}}\left[\left(a_{k^{\prime}}-\int d v_{\|} g_{k^{\prime}}, b_{k^{\prime}}\right)\left(\int d v_{\|} g_{k}, \hat{p}_{k} \frac{v_{\|}^{2}}{c^{2}}\right)\right. \\
& \left.\left.+\left(\int d v_{\|} g_{k}, b_{k}, \frac{v_{\|}}{c}\right)\left(\int d v_{\|} g_{k}, \hat{p}_{k} \frac{v_{\|}}{c}\right)\right]\right\} \\
& -\sum_{k^{\prime}+k^{\prime \prime}=k} \frac{T}{e}\left|w_{k^{\prime} k^{\prime \prime}}\right|^{2}\left\langle\tilde{A}_{\|}^{*} \tilde{q}\right\rangle_{k^{\prime \prime}} \\
& \times\left\{G_{k^{\prime}}, \frac{v \|}{c}\left[\left(a_{k^{\prime}}-\int d v_{\|} g_{k^{\prime}}, b_{k^{\prime}}\right)\left(\int d v^{\prime} \|_{k^{\prime}} \hat{p}_{k} \frac{v_{\|}^{2}}{c^{2}}\right)+\left(\int d v_{\|} g_{k^{\prime}}, b_{k}, \frac{v_{\|}}{c}\right)\left(\int d v_{\|} g_{k^{\prime}}, \hat{p}_{k} \frac{v_{\|}}{c}\right)\right]\right\}+\ldots .
\end{aligned}
$$

If we integrate this equation over $v_{\|}$at $k=k_{0}$, we see that the flux of perpendicular energy $\Gamma_{H_{\perp}}$ vanishes only when there is neither average temperature gradient, $\Delta T=0$, nor perpendicular temperature perturbation, $\delta T_{\perp k_{0}}=0$. The order of magnitude of this energy flux is obtained by considering the special case where $\Delta T=0$, which implies $\beta_{k^{\prime}}=0$ and $\tilde{q}_{k^{\prime \prime}}=0$. Then the only term which survives in Eq. (13) is the first one, which corresponds to the quasilinear estimate. 
In conclusion, we have shown that in spite of ambipolarity, there can be transport of both electron parallel and perpendicular energies, the latter being of the order of the test-particle diffusion. The transport of parallel energy is due to the contribution of the nonresonant electrons. The transport of perpendicular energy is due to a portion of the distribution function which is evolved only passively in the electromagnetic fields. The results obtained here have deep implications for transport studies in tokamak machines. Contrary to Terry, Diamond, and Hahm's assertion, ${ }^{3}$ the electron heat transport due to electromagnetic fluctuations is large, and is of the order of the test-particle diffusion in the turbulent field. It is clear from the expressions (12) and (13) that the equilibrium fluxes, obtained by integration of the $\Gamma$ 's, Eqs. (7)-(9), as the gradients are raised from zero to their equilibrium values, will be nonlinear functions of $T$ and of $\Delta T$.

We wish to thank Dr. J. A. Krommes for reporting to us his own work on this subject in advance of publication. This work was supported by U.S. Department of Energy Contract No. DE-FG02-84ER53174, and by the Cornell College of Engineering.
${ }^{1}$ See, for example, P. H. Rebut et al., in Proceedings of the Eleventh International Conference on Plasma Physics and Controlled Nuclear Fusion Research, Kyoto, Japan, 1986 (to be published), paper IAEA-CN-47/A-I-2.

${ }^{2}$ P. H. Rebut and P. P. Lallia, in Proceedings of the Second International Conference on Plasma Physics, Kiev, USSR, 1987 (to be published).

${ }^{3}$ P. W. Terry, P. H. Diamond, and T. S. Hahm, Phys. Rev. Lett. 57, 1899 (1986).

${ }^{4}$ A. B. Rechester and M. N. Rosenbluth, Phys. Rev. Lett. 40, 38 (1978).

${ }^{5}$ J. A. Krommes and C. B. Kim, Princeton Plasma Physics Laboratory Report No. PPPL-2473, 1987 (to be published); C. W. Horton, in Handbook of Plasma Physics, edited by A. A. Galeev and R. N. Sudan, Basic Plasma Physics Vol. 2 (North-Holland, Amsterdam, 1984), p. 413.

${ }^{6}$ J. A. Krommes, C. Oberman, and R. G. Kleva, J. Plasma Phys. 30, 11 (1983).

${ }^{7}$ B. B. Kadomtsev, Plasma Turbulence (Academic, New York, 1965).

${ }^{8}$ J. A. Krommes, in Handbook of Plasma Physics, edited by A. A. Galeev and R. N. Sudan, Basic Plasma Physics Vol. 2 (North-Holland, Amsterdam, 1984), p. 183.

${ }^{9}$ R. E. Waltz, Phys. Fluids 25, 1269 (1982). 